\title{
Development and Evaluation of Liposomes for Topical Delivery of Tacrolimus (Fk-506)
}

\author{
S. S. Patel ${ }^{\text {* }}$, M. S. Patel ${ }^{1}$, S. Salampure ${ }^{2}$, B. Vishwanath ${ }^{2}$, and N. M. Patel ${ }^{1}$ \\ ${ }^{1}$ Department of Pharmaceutical Technology, Shri B. M. Shah College of Pharmaceutical Education \\ and Research, Modasa-383315, Gujarat, India \\ ${ }^{2}$ Department of Pharmaceutics, Krupanidhi College of pharmacy, Bangalore, India
}

Received 5 September 2009, accepted in final revised form 9 August 2010

\begin{abstract}
In the present work Tacrolimus loaded liposomal systems were developed and evaluated for their topical delivery. Neutral multilamellar liposomes (MLVs) were prepared by thin film hydration method. The amount of drug loaded into vesicles ranged from $4.4 \mathrm{mg}$ per $115 \mathrm{mg}$ to $8.2 \mathrm{mg}$ per $140 \mathrm{mg}$ of total lipid. Entrapment efficiency of tacrolimus in liposomes was studied by altering the amount of cholesterol ratio to lipid ratio. After performing stability study at different temperatures $\left(4,25\right.$, and $\left.37^{\circ} \mathrm{C}\right)$ was affirms that drug leakage increased at higher temperature. The in-vitro permeation study shows significant reduced permeation with tacrolimus liposomes compared with free tacrolimus in propylene glycol. The animal study carried out on allergic contact dermatitis (ACD) model in rats showed that $0.03 \%$ tacrolimus liposomal gel exhibited similar activity when compared with $0.03 \%$ marketed tacrolimus ointment. It is also likely that tacrolimus liposomal gel which leads no visible or palpable residue when applied on skin would be more appealing to patients than conventional ointment. Overall study suggests that tacrolimus can be effectively incorporated in liposomes and can be used for the treatment of atopic dermatitis.
\end{abstract}

Keywords: Tacrolimus; Multilamellar vesicle; Allergic contact dermatitis.

(C) 2010 JSR Publications. ISSN: 2070-0237 (Print); 2070-0245 (Online). All rights reserved.

DOI: 10.3329/jsr.v2i3.3258 J. Sci. Res. 2 (3), 585-596 (2010)

\section{Introduction}

Tacrolimus, a macrolide immunosuppressant produced by Streptomyces tsukubaensis has been used since 1994 to prevent allograft rejection in liver and kidney transplants. Tacrolimus (TAC) is the first new topical therapy for the treatment of moderate to severe atopic dermatitis since the advent of topical corticosteroids [1]. The mechanism of action of tacrolimus in atopic dermatitis is not known. It has been demonstrated that tacrolimus inhibits T-lymphocyte activation by first binding to an intracellular protein, FKBP-12. Complex of tacrolimus-FKBP-12, calcium, calmodulin, and calcineurin is then formed

\footnotetext{
*Corresponding author: onlysanju2004@yahoo.co.in
} 
and the phosphatase activity of calcineurin is inhibited [2]. Tacrolimus is found to be effective in treating atopic dermatitis with main side effects like burning sensation of the skin, occurring in 48 to 58 percent treated with tacrolimus. Pruritis was reported in 46 percent of patients. With systemic tacrolimus, the principal adverse side effects are nephrotoxicity, neurotoxicity and gastrointestinal disturbances. Efficacy and safety of tacrolimus ointment compared with that of hydrocortisone butyrate ointment in adult patients with atopic dermatitis indicate that the efficacy of $0.1 \%$ tacrolimus ointment is comparable with that of $0.1 \%$ hydrocortisone butyrate ointment, a midpotent to potent topical corticosteroids used as standard therapy in AD. Treatment with tacrolimus ointment caused transient local burning and Pruritis. Folliculitis was common adverse effect prevalent in all 3 treatment group under clinical investigation [3]. Clinical trails data suggest some tacrolimus was absorbed, with levels in the blood stream reaching $4.7 \mathrm{mg}$ $\mathrm{mL}^{-1}$ in one patient using $0.3 \%$ ointment. This level in the blood stream probably exerts a subclinical effect on kidney function and may leave the patient vulnerable to the effect of interactions with concomitant drugs [4].

Liposomes are microscopic structures consisting of one or more concentric spheres of bilayers, enclosing aqueous compartments [5]. Several reports indicate that depending upon the nature (composition, size, surface charge), the drug and other ingredient present in liposomal product can fulfill both the requirements of system for localized drug effect as well as of drug carrier [6]. The liposomal formulations have been successful in treatment of a number of dermatological diseases [7].

The use of liposomes as drug carriers seems to have a promise for improved therapeutic prospects with a reduction of side effects [8]. Liposomal encapsulation of retenoids, e.g. vitamin A acid or tretinoin reduced the local irritation [9]. Data suggest that liposomal encapsulation of tacrolimus in topical formulation enhance drug penetration of skin. These characteristics may allow liposomal tacrolimus to be effective against psoriasis without the need of occlusive dressings. Should liposomal tacrolimus penetrate into the blood stream, experience in other models suggests it will be less toxic than free tacrolimus. This will be more important to patients who require higher concentrations of ointment to large areas of skin [4].

The study presented in this article includes the formulation of tacrolimus loaded liposomes by investigating the influence of various process parameters (film formation time, hydration time, temperature etc), morphological and micromeritic attributes, and drug leakage from vesicles. In vitro permeation study using rat abdominal skin to investigate the amount of drug released from formulation by topical route. Allergic contact dermatitis (ACD) model study on rats was used to investigate pharmacodynamic activity.

\section{Materials and Methods}

\subsection{Materials}

Tacrolimus monohydrate (FK-506) was a gift sample from Cadila Pharma Ltd (Ahemdabad India) and saturated soy lecithin (PC; lecichomax with 95\% phosphatidyl 
choline content) was a generous sample from Sonic Biochem (India). Cholesterol (CHOL), sephadex G-50 medium (bead size 50-150 $\mu \mathrm{m}$ ), butylated hydroxy toluene (BHT) and 2, 4-dinitro-1-flurobenzene (Sanger's reagent) were procured from Sigma Chemical Co. (USA). All other ingredients used in the study were HPLC or of analytical grade.

\subsection{Formulation of liposomes}

Multilamellar vesicles were prepared employing thin-film hydration technique using rotary flash evaporator (Laborota-4011 digital, heildolph, Germany). A lipid phase was prepared by dissolving accurately weighed quantities of the drug, soya phosphatidylcholine and cholesterol (Tables 1 and 2) in the chloroform-BHT (1:2\% $\mathrm{V} / \mathrm{V}$ ), in $250 \mathrm{ml}$ in round bottom flask containing glass beads. The solvent mixture was removed from the lipid phase by rotary evaporation at $45^{\circ} \mathrm{C}$ for 30 min under reduced pressure at $150 \mathrm{rpm}$ using rotary evaporator (Laborota 4011-Digital, Heidolph, Germany) in order to obtain a thin film of lipids on the wall of the flask and the surface of beads. Subsequently, the flask was kept under vacuum overnight to ensure the complete removal of residual solvent. The dry lipid film was hydrated with saline solution $(0.9 \% \mathrm{NaCl})$ at a

Table 1. Effect of drug-lipid ratio on the TAC loading in PC liposomes.

\begin{tabular}{cccc}
\hline $\begin{array}{c}\text { Batch } \\
\text { code }\end{array}$ & $\begin{array}{c}\text { TAC:PC:CHOL } \\
\text { (weight ratio in mg) }\end{array}$ & $\begin{array}{c}\text { Drug entrapped } \\
(\mathrm{mg} \pm \mathrm{SD})\end{array}$ & $\begin{array}{c}\text { Percent drug } \\
\text { loading }\end{array}$ \\
\hline F1 & $05: 100: 0$ & $2.61 \pm 0.11$ & 38.1 \\
F2 & $7.5: 100: 0$ & $3.41 \pm 0.15$ & 41.9 \\
F3 & $10: 100: 0$ & $4.92 \pm 0.21$ & 49.2 \\
F4 & $15: 100: 0$ & $4.62 \pm 0.16$ & 31.7 \\
\hline
\end{tabular}

*Value indicates mean \pm standard deviation $(n=5)$.

Table 2. Effect of drug-lipid ratio and amount of cholesterol on the TAC loading in PC liposomes.

\begin{tabular}{cccccc}
\hline $\begin{array}{c}\text { Batch } \\
\text { code }\end{array}$ & $\begin{array}{c}\text { TAC:PC:CHOL } \\
\text { (weight ratio in mg) }\end{array}$ & $\begin{array}{c}\text { Drug entrapped } \\
(\mathrm{mg} \pm \mathrm{SD})\end{array}$ & $\begin{array}{c}\text { Percent drug } \\
\text { loading }\end{array}$ & $\begin{array}{c}\text { Drug-lipid ratio } \\
(\mu \mathrm{g}: \mathrm{mg})\end{array}$ & $\begin{array}{c}\text { Mean particle } \\
\text { size }(\mu \mathrm{m})\end{array}$ \\
\hline TAC-1 & $10: 100: 5$ & $4.41 \pm 0.15$ & 44.1 & $42.0: 1$ & 1.209 \\
TAC-2 & $10: 100: 10$ & $6.42 \pm 0.21$ & 64.2 & $58.3: 1$ & 1.224 \\
TAC-3 & $10: 100: 20$ & $7.25 \pm 0.16$ & 72.5 & $60.4: 1$ & 1.346 \\
TAC-4 & $10: 100: 30$ & $8.26 \pm 0.14$ & 82.6 & $63.5: 1$ & 1.590 \\
TAC-5 & $10: 100: 40$ & $7.81 \pm 0.13$ & 78.1 & $55.7 ; 1$ & 1.967 \\
TAC-6 & $10: 100: 50$ & $7.76 \pm 0.14$ & 77.6 & $51.7: 1$ & 2.596 \\
TAC-7 & $10: 100: 60$ & $7.73 \pm 0.18$ & 77.3 & $48.3: 1$ & 3.578 \\
\hline
\end{tabular}

*Value indicates mean \pm standard deviation $(n=5)$. 
temperature of $45 \pm 2{ }^{\circ} \mathrm{C}$ for $30 \mathrm{~min}$. The dispersion thus obtained was vortexed for about 2 minutes. The dispersion was left undisturbed at room temperature for 2-3 hours to allow complete swelling of the lipid film and hence to obtain vesicular suspension. The finished liposomes were transferred into a suitable container and were stored in to the refrigerator.

\subsection{Drug entrapment studies}

Separation of unentrapped drug from the prepared liposomes was carried out by mini column centrifugation method [10]. Liposomal suspension $(0.1 \mathrm{ml})$ was placed in sephadex G-50 column (pre-saturated with empty liposomes) and centrifuged (R4C Laboratory Centrifuge, Remi Equipments Ltd, Mumbai) at $2000 \mathrm{rpm}$ for $3 \mathrm{~min}$. Elutes containing drug loaded liposomes were collected and observed under optical microscope (Optical invert microscopes, Lomo, USA) to ensure the absence of unentrapped drug particles. The vesicular suspension thus obtained was sonicated for $15 \mathrm{~min}$ in a bath sonicator. Two $\mathrm{ml}$ of ethanol was added to the vesicular suspension for complete release of drug from disturbed vesicles and volume was made up to $10 \mathrm{ml}$ with methanol. Quantitative estimation of tacrolimus was done using high performance liquid chromatography-mass spectroscopy (LCMS-2010A, Shimadzu, Japan). Acetonitrile: water (80:20 \% V/V) was used as mobile phase with Phenominex (Gemini) - ODS- $150 \mathrm{x}$ $4.6 \mathrm{~mm}, 5 \mu$ column. Elution mode was isocratic with flow rate $0.5 \mathrm{ml} / \mathrm{min}$ and injection volume was $10 \mu \mathrm{l}$ at ambient temperature. A solution of $1 \mu \mathrm{g}$ was made from the working stock $(100 \mu \mathrm{g} / \mathrm{ml})$ solution by appropriate dilution and $10 \mu \mathrm{l}$ of this solution was injected into LCMS system to determine the retention time (Fig. 1) and standard peak area.

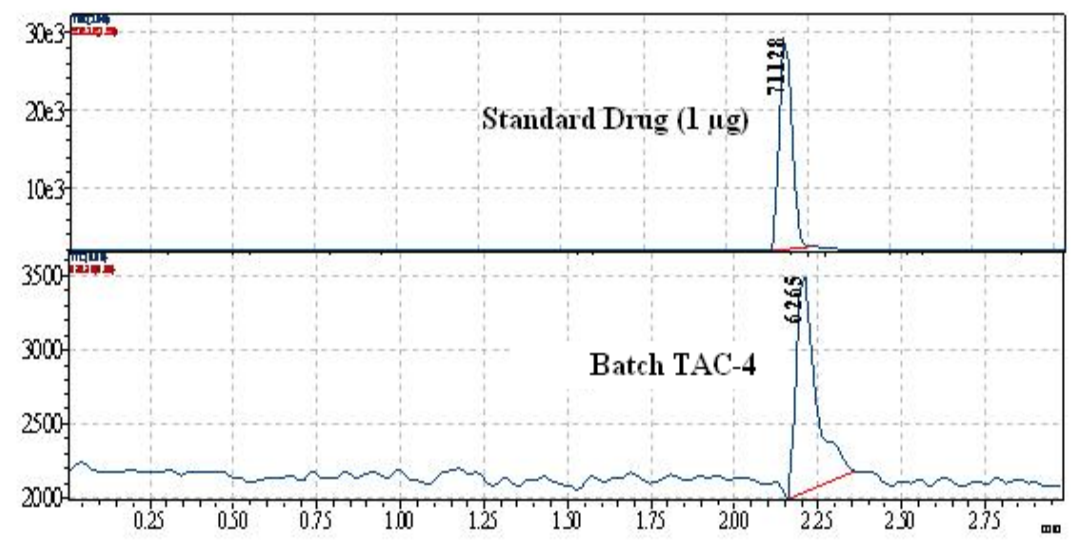

Fig. 1. Retention time of standard drug (conc. $1 \mu \mathrm{g}$ ) and sample TL-4.

The resulting peak area was compared with sample peak area to determine the sample concentration by following Eq. (1). 
Sample concentration $=\left(\frac{\text { sample area }}{\text { standard area }}\right) \times$ standard concentration

Percent drug loading (PDL) for the prepared liposomes were calculated from

$\mathrm{PDL}=\left(\frac{\text { Entrapped drug }(\mathrm{mg})}{\text { Total drug added }(\mathrm{mg})}\right) \times 100$

\subsection{Microscopy and size distribution profile}

Prepared liposomal batches were monitored for their morphological attributes using optical microscope (Optical invert microscopes, Lomo, USA) at suitable magnification. Size distribution profile of liposomes was determined by light scattering based on laser diffraction method employing Malvern Master-sizer (Hydro2000S, Malvern, UK).

\subsection{Storage-stability studies}

The ability of vesicles to retain the drug (i.e. drug retentive behavior) was assessed by keeping the liposomal suspensions at three different temperature conditions, i.e. $4-5^{\circ} \mathrm{C}$ (Refrigerator; RF), $25 \pm 2^{\circ} \mathrm{C}$ (Room temperature; RT) and $37 \pm 2^{\circ} \mathrm{C}$ for a period of 8 weeks [11]. The liposomal suspensions were kept in sealed vials (20 ml capacity) after flushing with nitrogen. Samples were withdrawn periodically and analyzed for drug content, in the manner described under drug entrapment studies.

\subsection{In-vitro skin permeation study}

Skin permeation studies with TAC containing liposomal formulations (liposome suspension and liposomes incorporated in carbopol gel) were carried out using hairless abdominal skin of Wister rat, employing modified Franz-diffusion cells. The results obtained were compared with that of non-liposomal formulation of tacrolimus, i.e. $1 \%$ TAC in propylene glycol, containing equivalent amounts of TAC. Briefly, to obtain skin, animals were sacrificed after getting approval of the animal ethics committee. Hair on the dorsal side of the animal was removed with the help of a $0.1 \mathrm{~mm}$ animal hair clipper, in the direction of tail to head. Dermis part of the skin was wiped 3 to 4 times with a wet cotton swab soaked in isopropranol to remove any adhering fat material. Skin was mounted on the receptor chamber with cross-sectional area of $3.15 \mathrm{~cm}^{2}$ exposed to the receptor compartment. Phosphate buffered saline solution: ethanol (3:1) (10 ml, $0.9 \%$ $\mathrm{w} / \mathrm{v}$ ) was employed as receptor phase and the temperature was maintained at $34 \pm 2^{\circ} \mathrm{C}$ [12]. Liposomal or non-liposomal TAC formulation (amount equivalent to $3 \mathrm{mg}$ of drug) was applied uniformly on the dorsal side of rat skin. Aliquots of $100 \mu \mathrm{L}$ were withdrawn periodically and replaced with same amount of saline solution to maintain the receptor 
phase volume at a constant level. The samples were quantified using LCMS method as described above.

\subsection{Evaluation of liposomal formulation in allergic contact dermatitis (ACD) model}

In this study the best formulation TAC-4 was incorporated into $1.5 \%$ carbopol gel base and compared with the marketed $0.03 \%$ tacrolimus ointment $(0.03 \%$ crolim ointment, Crosland's) in ACD model in rats [13]. Three groups of six animals were taken - one was used for control, the second group was used for standard and third one was used to study the activity of the test. To each group of control, standard and test was applied a blank carbopol gel (1.5\%), tacrolimus ointment (0.03\%) and tacrolimus liposomal gel (0.03 $\%)$, respectively on both ear pinna for seven days. On fifth day the rats from each group were sensitized with 2, 4-dintiro-1-flurobenzene dissolved in acetone and olive oil (4:1) onto the back. On the seventh day rats were challenged with 2, 4-dintro-1-flurobenzene (DNFB) on the pinna. Thickness of ear pinna was measured using digimatic vernier scale (Mutitoyo, Japan) before and after 24 hrs of challenge with DNFB. All values in case of ACD model in rat are presented as mean \pm SEM. Statistical significance was determined using ANOVA with Bonferroi’s test. $P<0.05$ was considered significant.

\section{Results and Discussion}

\subsection{Influence of process parameters on liposomal formulations}

Tacrolimus was chosen for encapsulation within the phospholipid bound closed lamellar systems in order to explore its potential for topical application. The encapsulated molecules of the active substance, in the Lipoidal polar-nonpolar folds of liposomes were expected to be delivered into the skin layers. Various product-influencing variables viz. vacuum, speed of rotation, hydration media and hydration time were studied in order to produce lipid vesicles of tacrolimus with desired attributes. Rotational speed of the flask demonstrated discernible influence on the thickness and uniformity of the lipid film. The optimum speed was noted to be $150 \mathrm{rpm}$, as the same yielded a uniform thin film on the flask and subsequently homogeneous population of liposomes. The film was kept under vacuum overnight to achieve complete drying and hence to avoid the formation of emulsion. The latter result may be due to the presence of residual organic solvent in the lipid film during hydration. Vortexing for two minutes was found to be appropriate to obtain the liposomal suspension free from aggregates. The process of vortexing did not affect the percent entrapment of drug in liposomes, which was confirmed by determining drug entrapment in liposomes, before and after vortexing. With regard to the influence of formulation components on the PDL, different compositions with varying ratios of drug, PC and CHOL (with $2 \%$ BHT, anti-oxidant) were studied. Table 1 summarizes the influence of drug-lipid ratio on the PDL of TAC in the liposomes. In case of CHOL free soy PC liposomes (batch F1 to F4), maximum drug loading of $49.2 \%$ could be achieved 
using 1:10 w/w drug-lipid ratio during preparation. The drug bearing capacity of liposomes (i.e. PDL) was found to be invariably dependent on drug-lipid ratio employed in the liposomal composition. A considerable enhancement in the PDL values was observed with increase in the amount of TAC from $5 \mathrm{mg}$ to $10 \mathrm{mg}$, i.e., up to drug-lipid ratio of 1:10 w/w (while keeping the amount of lipids fixed, i.e. $100 \mathrm{mg}$ ). However, the values of PDL tended to decrease with further increase in amount of the drug. A further improvement of 25 to $30 \%$ in the entrapment of TAC liposomes was noted with the addition of 5 to $40 \%$ w/w of CHOL (formulation TAC-1 to TAC-4, Table 2). Albeit the PDL was found to increase with CHOL addition, however, the effective drug-lipid ratio in the liposomes decreased due to increase in the total amount of lipids. The results revealed that the incorporation of CHOL enhanced the percent entrapment of TAC, owing to its cementing effect on the membrane packing. The same would prevent drug leakage from the bilayer membranes leading to enhanced drug retention in liposomes [14]. Based on the above findings, liposomal formulation TAC-4 was selected for further studies.

\subsection{Characterization of liposomes}

The size-range of all the formulations (TAC-1 to TAC-7) was found to be 1 to $9 \mu \mathrm{m}$, with $90 \%$ of the liposomal population equal or below $6.2 \mu \mathrm{m}$. The mean vesicle diameter of TAC-4 formulation was found to be $1.590 \mu \mathrm{m}$. Log-Size distribution curve (Fig. 2) confirms the normal size distribution of the vesicles. The reproducibility of the liposomal formulation with respect to size was confirmed by preparing the formulations five times, but the statistical analysis was avoided as the particle size data was highly reproducible each time. The optical photomicrograph of TAC-4 liposomes (Fig. 3) obtained at suitable magnification $(1000 \mathrm{x})$ confirms the multilamellar nature of the vesicles.

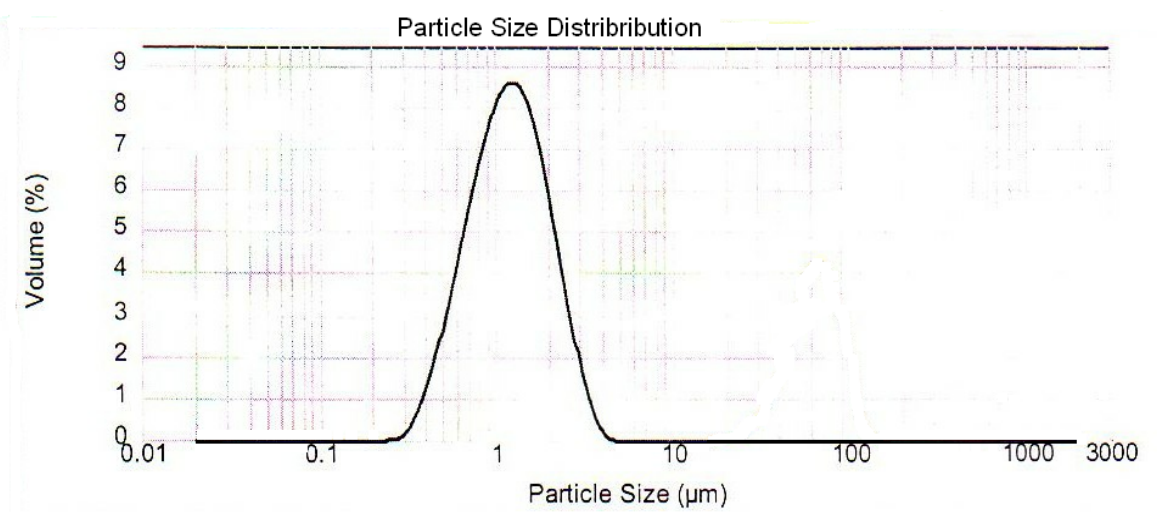

Fig. 2. Particle size distribution profile of liposomes formulation (TAC-4). 


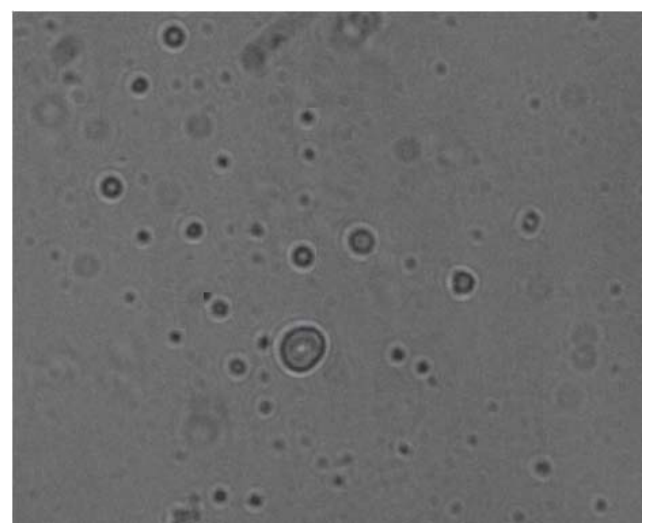

Fig. 3. Optical photomicrograph of tacrolimus loaded liposomes (TAC-4).

\subsection{Stability profile of liposomes}

TAC-4 liposomes were found to be reasonably stable in terms of aggregation, fusion and or vesicle disruption tendencies, over the studied storage period. Liposomes also retained their multilamellar nature and shape uniformity to an appreciable extent. The bar diagram (Fig. 4) depicts the percent drug leakage from liposomes over the eight weeks period, at

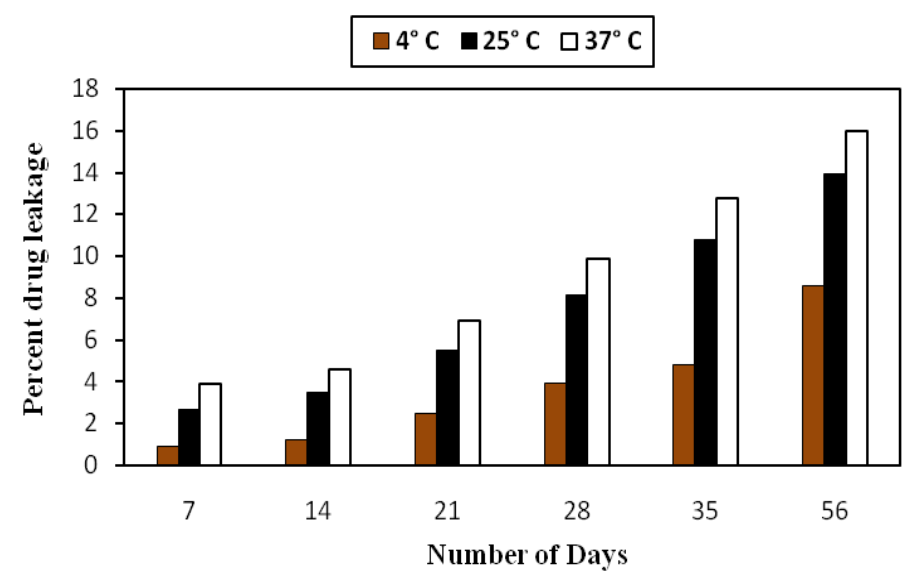

Fig. 4. Extent of drug leakage from TAC-4 liposomes after stability study.

at different storage temperatures. Substantial loss of drug, i.e. approximately $15.96 \%$ was evident from the samples stored at $37 \pm 2^{\circ} \mathrm{C}$ temperature. On the other hand, at lower temperature condition RF and $25 \pm 2{ }^{\circ} \mathrm{C}$, the liposomes could retain 91.44 and $86.12 \%$ of the incorporated drug, respectively. Acceleration in the drug leakage at higher temperatures, as observed in storage-stability studies, suggested keeping the liposomal 
product in the refrigeration conditions, to minimize the drug leakage from liposomalsystems. Loss of drug from the vesicles stored at elevated temperature may be attributed to the effect of temperature on the gel to liquid transition of lipid bilayers together with possible chemical degradation of the phospholipids, leading to defects in the membrane packing $[11,15]$. The drug leakage of less than $10 \%$ of the initial load at refrigeration conditions is well within the limits, when vesicles are to be advocated for topical applications.

\subsection{Skin permeation study}

Results obtained from in vitro drug permeation studies conducted with different formulations of TAC, are shown in Table 3. Significant augmentation in the skin permeation of tacrolimus has been observed (Fig. 5) with liposomal formulations vis-à-vis aqueous solution or carbopol gel. The amount of TAC permeated in 24 hrs was found to be $65.71 \%$ and $61.45 \%$ from liposomal suspension and liposomal gel, respectively,

Table 3. In vitro skin permeation of tacrolimus from different formulations.

\begin{tabular}{lcc}
\hline \multicolumn{1}{c}{ TAC Formulations } & $\begin{array}{c}\text { Mean cumulative percent drug } \\
\text { permeated } \pm \text { S.D. }\end{array}$ & $\begin{array}{c}\text { Permeation flux } \\
\left(\mathrm{ng} / \mathrm{cm}^{2} / \mathrm{hr}\right)\end{array}$ \\
\hline Aqueous solution & $49.57 \pm 1.90$ & 0.932 \\
Drug in carbopol gel & $35.21 \pm 2.13$ & 1.237 \\
Liposomal suspension & $65.71 \pm 3.48$ & 2.623 \\
Liposomal gel & $61.45 \pm 3.11$ & 1.740 \\
\hline
\end{tabular}

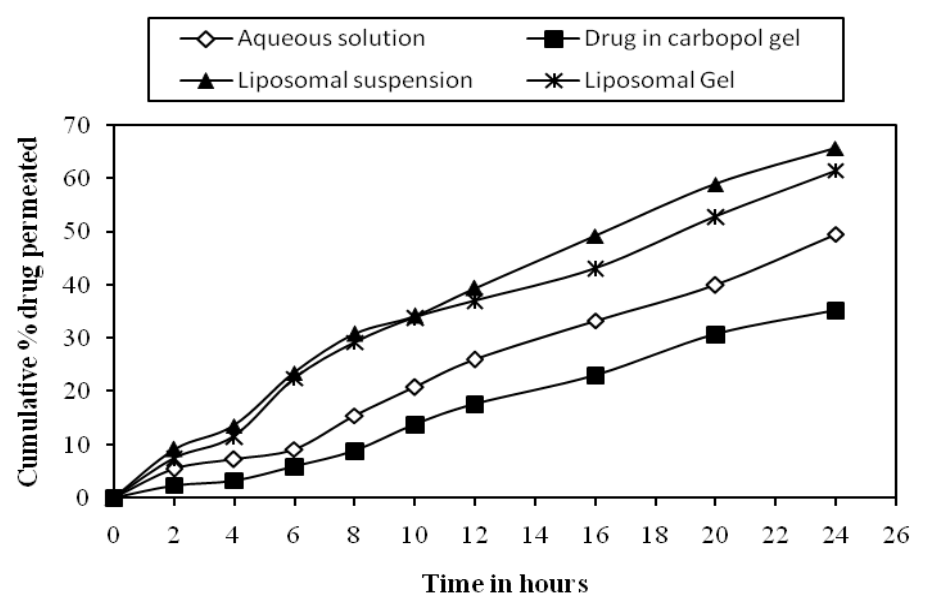

Fig. 5. Permeation profile of tacrolimus containing systems across rat skin. 
whereas only $49.57 \%$ and $35.21 \%$ of the drug permeated in case of aqueous solution and carbopol gel, respectively. Higher values of flux obtained with liposomal suspension $\left(2.623 \mathrm{ng} / \mathrm{cm}^{2} / \mathrm{hr}\right)$, and liposomal gel $\left(1.740 \mathrm{ng} / \mathrm{cm}^{2} / \mathrm{hr}\right)$, than those obtained with aqueous solution $\left(0.932 \mathrm{ng} / \mathrm{cm}^{2} / \mathrm{hr}\right)$ and carbopol gel $\left(1.237 \mathrm{ng} / \mathrm{cm}^{2} / \mathrm{hr}\right)$ clearly vouch for the permeation enhancing effect of vesiculation on the drug. The liposomal system composed of phospholipids (also one of the natural constituent of skin lipids) helped generating and retaining the required physico-chemical state of the skin for enhanced permeation of the tacrolimus. Further, the phospholipid-rich domains of vesicles might have helped to produce the depot effect for drug molecules. The latter has been reflected as higher amount of drug retention within skin layers in case of liposomal formulations.

\subsection{Clinical trial of the developed formulation (ACD model)}

Figs. 6 and 7 indicate the ear thickness in right and left ear, respectively after application of $1.5 \%$ blank carbopol gel, marketed $0.03 \%$ tacrolimus ointment and $0.03 \%$ liposomal

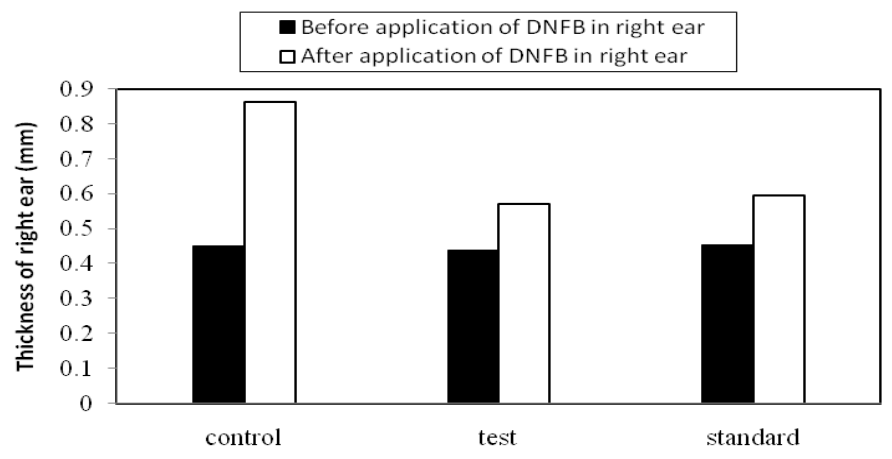

Fig. 6. Effect of different tacrolimus systems in right ear.

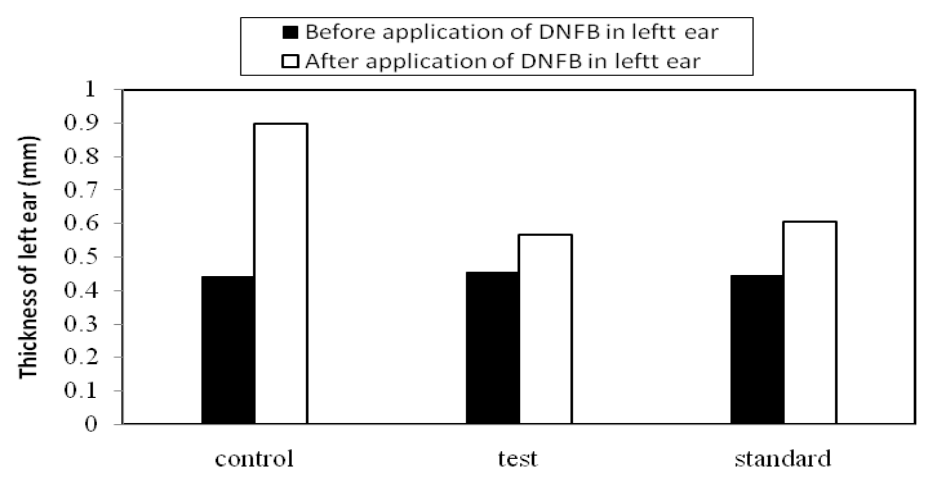

Fig. 7. Effect of different tacrolimus systems in leftt ear. 
gel TAC-4 in control, standard and test group respectively. Before the application of DNFB on ear pinna to 3 groups (i.e. control, standard and test) no significant difference in thickness of ear pinna was found. After the application of DNFB in 3 said groups, significant decrease in ear thickness in test group was found as compared with control group. On the contrary no significant difference was observed when test was compared with standard group. The result obtained shows that $0.03 \%$ tacrolimus liposome was effective in allergic contact dermatitis model in rats with similar efficacy as of $0.03 \%$ marketed tacrolimus ointment in the same model.

\section{Conclusions}

Tacrolimus (FK-506) could be successfully entrapped in the liposomes with reasonable drug loading and desired vesicle specific characters. Higher rate of drug transfer across the skin with liposomal formulations of tacrolimus suggests that the drug in its liposolubilised state might have found facilitated entry into the tough barrier consisting of stratum corneum. The amount of drug loaded into vesicles ranged from $4.4 \mathrm{mg}$ per $115 \mathrm{mg}$ to $8.2 \mathrm{mg}$ per $140 \mathrm{mg}$ of total lipid. Through skin permeation studies it can be predicted that percutaneous absorption of tacrolimus liposomes into systemic circulation after topical application to patient will be significantly less. The flux value obtained was very low i.e. $1.740 \mathrm{ng} / \mathrm{hr} / \mathrm{cm}^{2}$ for tacrolimus liposomes as compared with $2.623 \mathrm{ng} / \mathrm{hr} / \mathrm{cm}^{2}$ of tacrolimus in propylene glycol. Animal study data suggest that $0.03 \%$ tacrolimus liposomal gel showed similar activity when compared with $0.03 \%$ marketed tacrolimus ointment. It is also likely that tacrolimus liposomal gel, which leads no visible or palpable residue when applied to skin, will be more appealing to patients than the conventional ointment. Clinical trials comparing commercial tacrolimus ointment with developed tacrolimus liposomes are required to test the safety and efficacy in humans.The results obtained in the study suggest that tacrolimus can be effectively incorporated in liposomes and can be used for the treatment of atopic dermatitis.

\section{Acknowledgement}

We are thankful to Cadila Pharmaceutical, Ahmedabad, India and Sonic Biochem, India, for generously providing the gift samples of Tacrolimus (FK-506) and Lecithin, respectively. Our thanks are also due to Research and Development, Strides Arcolabs Ltd. Bangalore, India, for providing facilities for carrying out the research work.

\section{References}

1. J. Russel, Am. Fam. Physician. 66, 1899 (2002).

2. Martindale, $33^{\text {rd }}$ edition. (Pharmaceutical press, New York, 2002).

3. S. Reitamo, R. Malcolm, R. Thomas, C. Frederic, and K. Kirsti, J. Allergy. Clin. Immunal. 109, 547 (2002). doi:10.1067/mai.2002.121832

4. M. J. Erdogan, J. Wright, and V. McAlister, Br. J. Dermatol. 146, 964 (2002). doi:10.1046/j.1365-2133.2002.04800.x 
5. A. D. Bangham, M. M. Standish, and J. C. Watkin, J. Mol. Biol. 13, 238 (1965). doi:10.1016/S0022-2836(65)80093-6

6. A. Blume, Liposome Dermatics 11, 29 (1993).

7. R. Singh, and S. P. Vyas, Ind. J. Pharm. Sci. 58, 9 (1996).

8. W. Wohlrab, J. Lasch, R. Laub, C. M. Taube, and K. Wellner, Liposome Dermatics. 12, 215 (1993).

9. M. Mezi, Liposome Dermatics 12, 206 (1993).

10. S. R. Dipali, S. B. Kulkarni, and G. V. Betagiri, J. Pharm. Pharmacol. 48, 1112 (1996).

11. S. B. Tiwari, N. Udupa, B. S. S. Rao, and D. Puma, Int. J. Pharm. 32, 214 (2000).

12. A. Billich, A. Heinrich, A. Andras, and S. Anton, Int. J. Pharm. 269, 29 (2004). doi:10.1016/j.ijpharm.2003.07.013

13. T. Katafuchi, T. Kondo, and T. Hori, Neuroimmunomodulation 9, 157 (2001).

14. R. Aggarwal, and O. P. Katare, Pharm. Tech. 26, 48 (2002).

15. R. Agrawal, O. P. Katare, and S. P. Vyas, Int. J. Pharm. 228, 43 (2001). 